\title{
A Case with the Bilateral Narrow Bony Cochlear Nerve Canals Associated with Near Normal Hearing Thresholds
}

\author{
Jae-Cheul Ahn ${ }^{1}$, Shin-Hye Kim ${ }^{1}$ and Byung Yoon $\mathrm{Choi}^{2}$ \\ ${ }^{1}$ Department of Otorhinolaryngology-Head \& Neck Surgery, Seoul National University Hospital, Seoul, \\ ${ }^{2}$ Department of Otorhinolaryngology-Head \& Neck Surgery, Seoul National University Bundang Hospital, Seongnam, Korea
}

Received September 13, 2012

Revised October 18, 2012

Accepted October 20, 2012

\author{
Address for correspondence \\ Byung Yoon Choi, MD \\ Department of Otorhinolaryngology- \\ Head \& Neck Surgery, \\ Seoul National University \\ Bundang Hospital, \\ 82 Gumi-ro 173beon-gil, \\ Bundang-gu, Seongnam 463-707, \\ Korea \\ Tel +82-31-787-7406 \\ Fax +82-31-787-4057 \\ E-mail choiby@snubh.org
}

\begin{abstract}
The narrow bony cochlear nerve canal in high resolution temporal bone computed tomography is frequently found in patients of congenital sensorineural hearing loss. But this bony structural anomaly could not conclude the functional outcome of cochlear nerve. Here, we present a case of a 14-month-old girl having bilateral narrow bony cochlear nerve canals. In magnetic resonance imaging, both the cochlear nerves were identified to be intact. Moreover, acoustic brainstem response threshold and auditory steady state response revealed nearly normal hearing thresholds. Therefore, we suggest that the narrow bony cochlear nerve canal itself does not necessarily indicate a substantial degree of hearing loss.
\end{abstract}

Korean J Audiol 2012;16:141-144

KEY WORDS: Cochlear nerve · Multidetector computed tomography · Sensorineural hearing loss $\cdot$ Magnetic resonance imaging.

\section{Introduction}

With advancement of a radiologic imaging technique, more information can be obtained from the imaging and it would give a hand to diagnosis and decision making for treatment. High resolution temporal bone computed tomography (TBCT) and internal auditory canal magnetic resonance image (IAC MRI) provide detailed images of inner ear and cochleovestibular nerve. High resolution TBCT is a tool commonly used to evaluate structures of the middle ear and inner ear when the structural deformities or anomalies are clinically suspected. Recently, narrow bony cochlear nerve canal (BCNC) is identified and attracts a lot of attention. ${ }^{1,2)}$ The $\mathrm{BCNC}$ is the short canal between the base of the cochlear modiolus and the fundus of the internal auditory canal (IAC) carrying the cochlear nerve fibers from spiral ganglion to cochlear nucleus. ${ }^{3)} \mathrm{Nar}-$ row $\mathrm{BCNC}$ is considered when the width is less than $1.4 \mathrm{~mm}$ to $1.5 \mathrm{~mm}^{3}{ }^{3}$

Some sensorineural hearing loss (SNHL) patients show narrow BCNC or narrow IAC in TBCT or IAC MRI. ${ }^{1,45)}$ Furthermore, both the $\mathrm{BCNC}$ and IAC are frequently narrower in the affected side of unilateral SNHL. ${ }^{6,7)}$ In other way, cochlear ner- ve deficiency (CND) is shown in the substantial proportion of narrow BCNC cases. ${ }^{8}{ }^{89)}$ Therefore, it can be deduced that the narrow $\mathrm{BCNC}$ as detected by the TBCT would play some role in the SNHL.

However, it is noteworthy that the intact cochlear nerve is frequently visualized by IAC MRI in the bilateral narrow BCNC cases ${ }^{5,10)}$ contradicting the previous studies that the CND coincides with the narrow BCNC. ${ }^{8,911)}$ Therefore, we cannot conclude that the absence or hypoplasia of cochlear nerve necessarily accompanies the narrow $\mathrm{BCNC}$ especially in bilateral cases. However, there is a recent case report for the narrow $\mathrm{BCNC}$ not accompanied by profound hearing loss. ${ }^{12)}$ In the report, auditory brainstem response (ABR) was not detected even though only mild hearing loss was documented in pure tone audiometry (PTA). ${ }^{12)}$ It shows that narrow $\mathrm{BCNC}$ does not always lead to profoundly elevated pure tone thresholds. However, the absence of ABR responses implied the broken integrity of the cochlear nerve, which was in line with the CND shown in IAC MRI.

Here, we report a case with the bilateral narrow $\mathrm{BCNC}$ but with the intact cochlear nerve and nearly normal ABR thresholds. 


\section{Case Report}

A female newborn was referred to department of otorhinolaryngology in a tertiary hospital for evaluation of congenital both ear pits. She had intra-partum and post-partum medical problems such as low birth weight, intrauterine growth retardation, ventriculomegaly, fetal hydronephrosis, umbilical hernia, and craniofacial anomaly.

On physical examination, she had cup ear deformities, depressed helix, and both preauricular fistulae in both auricles. But her both tympanic membranes were normal. In newborn hearing screening automated acoustic brainstem response (AABR) performed with AccuScreen (GN Otometrics, Copenhagen, Denmark), both ear were reported as "refer". Then, acoustic brainstem response threshold (ABRT) and auditory steady state response (ASSR) were performed with Navigator PRO system and MASTER system (Bio-logic, San Carlos, CA, USA) and they were checked at the age of 2 months and the results were that right and left ABRT was $25 \mathrm{~dB}$ and 30 $\mathrm{dB}$, respectively and right and left ASSR were $25 \mathrm{~dB}$ and 30 $\mathrm{dB}$ in average, respectively (Fig. 1).

The $0.7 \mathrm{~mm}$ thickness high resolution TBCT performed with Philips Brilliance computed tomography 64-channel scanner (Philips Medical Systems, Cleveland, OH, USA) showed the significantly narrow BCNC (Fig. 2) in which the right and left diameters were $0.6 \mathrm{~mm}$ and $0.9 \mathrm{~mm}$, respectively (the right $0.6 \mathrm{~mm}$ and left $0.9 \mathrm{~mm}$ diameters have accuracy $42.9 \%$ and $64.3 \%$ in the $0.7 \mathrm{~mm}$ thickness images but both diameters are less than $1.4 \mathrm{~mm}$, the cutoff diameter of BC-NC, in $100 \%$ accuracy).

At the age of 14 months, distortion product otoacoustic emission (DPOAE) was performed with ILO (Otodynamics, Hatfield, UK) and IAC MRI was performed with a Philips 3.0 T Achieva (Philips Medical Systems, Best, the Netherlands). There were no response of DPOAE in right ear and small response of DPOAE only in left ear. This time she had effusion on right tympanic membrane.

The cochlear nerve in high resolution T2 weighted IAC MRI and constructive inference in steady state (CISS) MRI revealed an intact cochlear nerve in both sides (Fig. 3). No vestibular symptom, such as recurrent vomiting, abnormal gait or fall-down, and spontaneous nystagmus have been reported.

\section{Discussion}

Congenital sensorineural hearing loss is associated with bony abnormalities of the inner ear visible on CT in only $20 \%$ of cases. ${ }^{13,14)} \mathrm{Up}$ to recently, narrow $\mathrm{BCNC}$ has been considered one of the abnormalities which might easily explain the CND and resultantly, SNHL. However, narrow BCNC does

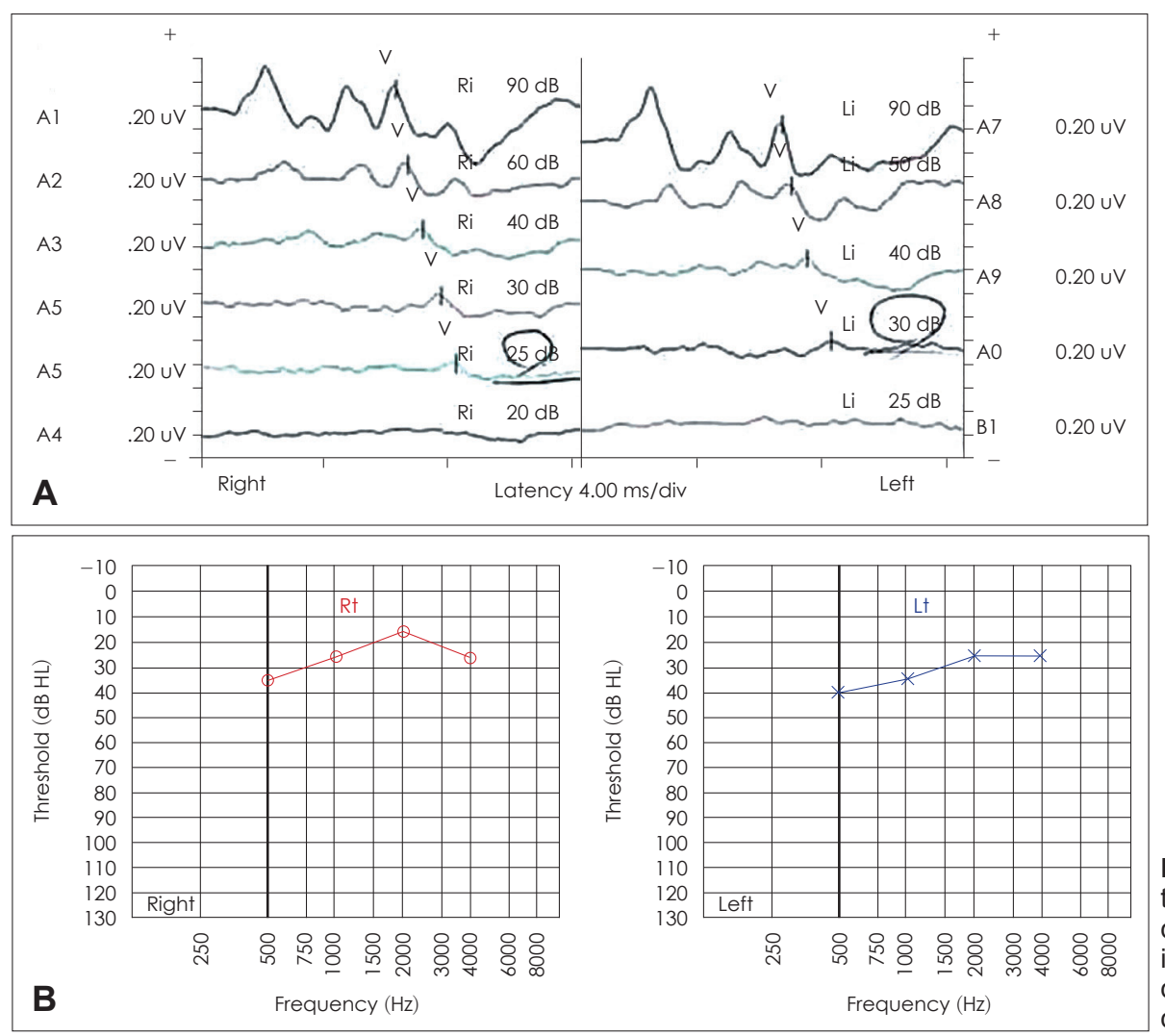

Fig. 1. Acoustic brainstem response threshold (ABRT) and auditory steady state response (ASSR). A: ABRT illustrated right $25 \mathrm{~dB} H \mathrm{HL}$ and left 30 $\mathrm{dB}$ HL. B: ASSR illustrated right 25 $\mathrm{dB} H \mathrm{HL}$ and left $30 \mathrm{~dB} \mathrm{HL}$. 
Fig. 2. Axial section of TBCT of the case patient and the control patient who is a 8 month-old girl with congenital bilateral sensorineural hearing loss and normal bony cochlear nerve canal width (field of view: $100 \times 100$ $\mathrm{mm}$, window width: 4000 , window level: 300 , thickness: $0.7 \mathrm{~mm})$. A: The width of right $\mathrm{BCNC}$ (between black arrows) was $0.6 \mathrm{~mm}$ in the case patient. B: The width of left BCNC was $0.9 \mathrm{~mm}$ in the case patient. C: The width of right $\mathrm{BCNC}$ was $1.7 \mathrm{~mm}$ in the control patient. $D$ : The width of left $\mathrm{BCNC}$ was $1.6 \mathrm{~mm}$ in the control patient. TBCT: temporal bone computed tomography, BCNC: bony cochlear nerve canal.

Fig. 3. Parasagittal constructive interference in steady state MRI (field of view: $160 \times 160 \mathrm{~mm}$, window width: 600 , window level: 280 , thickness: $0.6 \mathrm{~mm}$ ). Parasagittal CISS image was achieved by perpendicular plane to the internal auditory canal in both coronal and axial images. A: Right cochlear nerve entered into cochlear basal turn. B: Right cochlear nerve was identified in IAC. C: Left cochlear nerve entered into cochlear basal turn. D: Left cochlear nerve was identified in IAC. CN: cochlear nerve, FN facial nerve, SVN: superior vestibular nerve, IVN: inferior vestibular nerve, CISS: constructive inference in steady state, IAC: internal auditory canal.
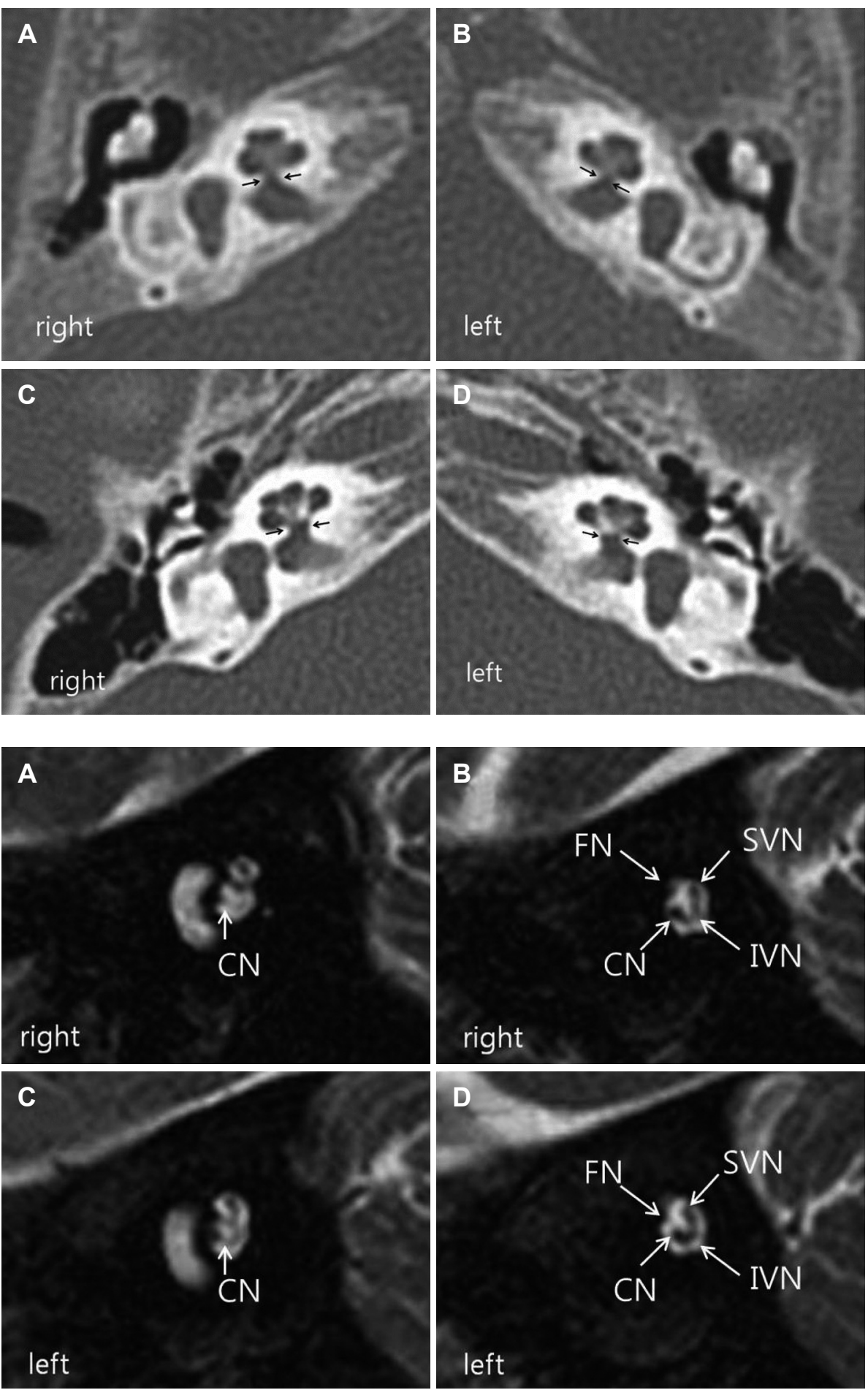

not necessarily associate morphological aplasia or hypoplasia of cochlear nerve in IAC MRI and CISS images. ${ }^{5,10)}$ Most of the narrow BCNC cases without CND have also been accompanied by severe or profound hearing thresholds. Further, it has been recently reported that the narrow $\mathrm{BCNC}$ is not always accompanied by significant SNHL and sometimes can be associated with only subtle hearing loss even under the in- visible cochlear nerve by MRI and poor ABR responses. ${ }^{12)}$ However, the narrow BCNC case without both CND and hearing loss has rarely been reported in the literature. In this study, we clearly present a narrow $\mathrm{BCNC}$ case where there is neither significant hearing loss nor the presence of CND, showing that we can suspect this anomaly even under near normal hearing status. 
In our study, the patient underwent AABR one time and had "REFER" sign in both ears. At that time, both tympanic membranes were free of pathologic findings. AABR have been conducted in newborn hearing screening with high referring rate $6.5 \%{ }^{15)}$ Considering the high referring rate of AABR, near normal results of ABRT and ASSR shows that AABR was false positive. The following DPOAE in 14-month-old revealed no response on the right ear. It was also false positive result of DPOAE since the right tympanic membrane showed effusion under the otoscope examination. Therefore, the near normal electrophysiologic audiometric results in this case suggested that we should include narrow BCNC as one of differential diagnosis even in subtle to mild SNHL.

The narrower BCNC width $0.6 \mathrm{~mm}$ on right ear of the case seemed to oppose the better ABRT and ASSR result $25 \mathrm{~dB}$ $\mathrm{HL}$ on right ear. But the cross section of $\mathrm{BCNC}$ must not have an exact circle. It can be a ovoid or irregular. With the variety of the sectional shape of BCNC, considering accuracy of the BCNC width under $0.7 \mathrm{~mm}$ thickness TBCT, $0.6 \mathrm{~mm}$ and 0.9 $\mathrm{mm}$ on the axial-plane images could not conclude the 3 -dimentional size difference. In other words, the size less than 1.4 $\mathrm{mm}$ under the $0.7 \mathrm{~mm}$ thickness axial-plane images can be confirmed with $100 \%$ accuracy but the size difference less than $1.4 \mathrm{~mm}$ cannot be concluded. In the case, the right BCNC might not be smaller than the left one.

The limitation of this case report is that only electrophysiological hearing tests were conducted such as AABR, ABRT, ASSR and DPOAE without PTA and speech audiometry (SA). So far, we could verify her hearing level with only electrophysiologic tests. The direct hearing tests, such as PTA and SA, are required to evaluate her hearing in near future.

There were many reports about morphological integrity of cochlear nerve in narrow BCNC. Also there was a few reports about mild hearing loss in narrow the BCNC. Given this, this is the report suggesting that a phenotypic spectrum of the narrow $\mathrm{BCNC}$ may range to the intact cochlear nerve and the near normal auditory threshold. The narrow BCNC should also be included as a differential diagnosis when a subtle hearing loss is encountered.

\section{Acknowledgments}

This study was supported by the Seoul National University Bundang Hospital research fund [No. 04-2010-003 (to Choi, B.Y)].

\section{REFERENCES}

1) Fatterpekar GM, Mukherji SK, Alley J, Lin Y, Castillo M. Hypoplasia of the bony canal for the cochlear nerve in patients with congenital sensorineural hearing loss: initial observations. Radiology 2000; 215:243-6.

2) Simons JP, Mandell DL, Arjmand EM. Computed tomography and magnetic resonance imaging in pediatric unilateral and asymmetric sensorineural hearing loss. Arch Otolaryngol Head Neck Surg 2006; 132:186-92.

3) Stjernholm C, Muren C. Dimensions of the cochlear nerve canal: a radioanatomic investigation. Acta Otolaryngol 2002;122:43-8.

4) Teissier N, Van Den Abbeele T, Sebag G, Elmaleh-Berges M. Computed Tomography measurements of the normal and the pathologic cochlea in children. Pediatr Radiol 2010;40:275-83.

5) Adunka OF, Roush PA, Teagle HF, Brown CJ, Zdanski CJ, Jewells $\mathrm{V}$, et al. Internal auditory canal morphology in children with cochlear nerve deficiency. Otol Neurotol 2006;27:793-801.

6) Kono T. Computed tomographic features of the bony canal of the cochlear nerve in pediatric patients with unilateral sensorineural hearing loss. Radiat Med 2008;26:115-9.

7) Shiomi Y, Shiomi Y, Oda N. Hearing impairment in a patient with unilateral narrowing of the internal auditory canal--a case report. Auris Nasus Larynx 2001;28:353-5.

8) Komatsubara S, Haruta A, Nagano Y, Kodama T. Evaluation of cochlear nerve imaging in severe congenital sensorineural hearing loss. ORL J Otorhinolaryngol Relat Spec 2007;69:198-202.

9) Morimoto N, Miyasaka M, Iigaya N, Matsuda A, Taiji H. Clinical aspects of unilateral hearing loss due to isolated cochlear nerve aplasia. Otol Jan 2009;19:41-8.

10) Adunka OF, Jewells V, Buchman CA. Value of computed tomography in the evaluation of children with cochlear nerve deficiency. Otol Neurotol 2007;28:597-604.

11) Choi YJ, Park SY, Kim MS, Sung KJ. The significance of a hypoplastic bony canal for the cochlear nerve in patients with sensorineural hearing loss: CT and MRI findings. J Korean Radiol Soc 2004;50: 227-36.

12) Miyanohara I, Miyashita K, Takumi K, Nakajo M, Kurono Y. A case of cochlear nerve deficiency without profound sensorineural hearing loss. Otol Neurotol 2011;32:529-32.

13) Winslow CP, Lepore ML. Imaging quiz case 1. Bilateral agenesis of lateral semicircular canals with hypoplasia of the left internal auditory canal (IAC). Arch Otolaryngol Head Neck Surg 1997;123:1236, 1238-9.

14) Jackler RK, Luxford WM, House WF. Congenital malformations of the inner ear: a classification based on embryogenesis. Laryngoscope 1987;97(3 Pt 2 Suppl 40):2-14.

15) Olusanya BO, Bamigboye BA. Is discordance in TEOAE and AABR outcomes predictable in newborns? Int J Pediatr Otorhinolaryngol 2010;74:1303-9. 